\title{
«La Chimie en Couleurs»: Socially Relevant \& Original Research in Chemistry in High Schools Using Modest Resources
}

\author{
Thibaud Rossel $\left.\right|^{\text {sa* }}$ and Marc Creus ${ }^{\mathrm{b}}$
}

§Balmer Prize 2018

\begin{abstract}
Teaching chemistry at high school level has the potential of playing a major role for the development of our society, in particular, to form future leaders in chemistry who will address social challenges such as the need for better healthcare, improved agricultural techniques and more efficient use of energy resources. In general, high school chemistry teaching programs tend to illustrate the great historic discoveries and glorious past of chemical research. It is hoped that this historical perspective will help to provide students with the basic understanding necessary for the development of the chemistry of tomorrow. Unfortunately, in general, the emphasis on established chemical research and on the reassuringly solid foundations of the field is ubiquitous, not only in the theoretical classes, but also in more practical aspects of teaching, such as in the 'maturity projects' of students: These small, often laboratory-oriented 'research projects' are generally limited to reproducing the scientific literature - often printed in black \& white - and/or are adding minor modifications to established scientific protocols, instead of exploring the colourful world of current scientific discoveries and the excitement of pushing back the boundaries of knowledge. Practicing innovative and original research with chemistry students is therefore a challenge for the mentor of any 'maturity project'. Here, we describe the implementation of a practical program - nicknamed 'La Chimie en Couleurs' - for carrying out original research in chemistry, making science lively, colourful and vivid to students. Science that has not already been done by others before, but that students can pursue themselves and that is totally new and original. The program is taught during high-school courses and carried out by students, using inexpensive equipment, easily accessible and non-toxic chemicals and simple chemical concepts. Part of the research work was presented by students at the Swiss Chemical Society Fall Meeting and won a poster prize in the inorganic chemistry runner up category (2016). The 'La Chimie en Couleurs' program presented here shows that up-to-date and socially-relevant chemistry (not just historically relevant chemistry!) can be taught to teenagers in a creative way through the implementation of inexpensive, albeit serious, scientific research at high school level.
\end{abstract}

Keywords: Combinatorial chemistry · Education · Indicator displacement assays · Inorganic chemistry · Research

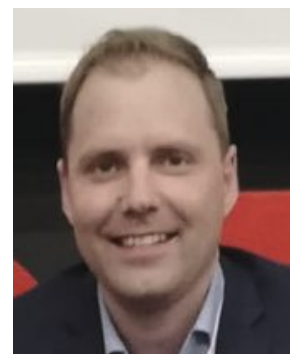

Dr. Thibaud Rossel studied biology at the University of Neuchâtel where he obtained a master degree in 2006. He then moved to the University of Basel where he obtained a $\mathrm{PhD}$ under the supervision of Prof. Dr. Thomas. R. Ward in the field of bio-inorganic chemistry (2011). He later took a position at the Gymnase français de Bienne (2013) where he teaches biology and chemistry. He was awarded the SCS Balmer prize in 2018 for his work with students in research and education at the interface of biology and chemistry related to optical biosensors against socially interesting analytes (such as phosphate or glyphosate) as well as the EPFL LEARN award $\left(3^{\text {rd }}\right)$ in 2019.

\section{General Motivation}

The main motivation for setting up this teaching program arose from the conviction that science is much more than an accumulation of past facts and knowledge. One should not restrict teaching science to simply enumerate the great discoveries of the past ('black \& white science'), but also to transmit the vision that science is a current, living, ongoing and colourful pursuit of knowledge. Science is not just what is known, but also the process of discovering what is not yet known, which requires freedom to explore. The aim of 'La Chimie en Couleurs' is to transmit the passion for research to students by guiding and facilitating original exploration in chemistry, in a way that is not only accessible, but also exciting.

\section{General Scientific Background and Experiments}

Chemistry has naturally divided into several fields of research such as organic chemistry, ${ }^{[1]}$ inorganic chemistry[2] and physical chemistry. Each of these fields use their own languages and have their own research methodologies. Here, we decided to focus our teaching in the field of inorganic chemistry, for two main reasons: i) because it is an important field with many applications that can be discussed with students, such as asymmetric catalysis, which gave birth to different Nobel prizes, ${ }^{[3]}$ and ii) because we consider 
that inorganic chemistry offers the advantage of simple methodological implementation with very little equipment necessary for experiments. Specifically, we decided to pursue the exciting field of construction of inorganic complexes, ${ }^{[4]}$ particularly by using reactions that occur in mild conditions, even in exposure to air and in water as solvent. An additional advantage is that the creation of inorganic complexes opens up research to combinatorial chemistry ${ }^{[5]}$ by varying ligands and metals. A library of inorganic complexes can thus be formed. This library of complexes can be scrutinized for different applications, such as catalysis or also for the detection of different molecules e.g. phosphates. ${ }^{[6]}$ This shuffling leads to the possibility of combinatorial or high-throughput screening. Hence, rounds of screenings allow to optimize the library through directed evolution protocols, for example. ${ }^{[7]}$ The detection of small molecules is fundamental in several research areas such as cell signalling or water quality. ${ }^{[7]} \mathrm{A}$ rapidly evolving domain is the recognition via inorganic complexes for the detection of anions. This application is useful for tracing ions in the cell, for example, or for detecting the presence of pollutants, and is therefore of major interest for research in medicinal diagnostics, environmental monitoring or wastewater treatment. ${ }^{[7]}$ Various research groups have developed anion detectors, ${ }^{[8,9]}$ most notably phosphate detectors based on inorganic complexes bearing copper or zinc as metals. ${ }^{[6,10]}$ These sensors have the advantage of being easily formed by successively mixing the ligand, the met$\mathrm{al}$, and the corresponding anion in a $\mathrm{pH}$-defined buffer solution. Detection is based on the indicator displacement assay (IDA) principle; therefore, it is colorimetric and visible to the naked eye (see Fig. 1). ${ }^{[11]}$ In its succession, the formation of inorganic complexes and the detection of anions via the IDA system present few major technical and scientific difficulties. It requires basic knowledge of organic chemistry, bases of inorganic chemistry, calculations of concentrations and simple principles of spectrophotometry. These are skills that students at high school acquire during their training and which can be easily complemented by the mentor of the maturity project. Students are given the task of mixing ligands, metal precursors, and detectors in buffers to form heteroleptic complexes and the task of investigating a range of analytes to detect them. If 'hits' are obtained, i.e. combinations leading to a change in colour upon target-binding, they are investigated in further detail and applications. Ultimately, this research may even be submitted for publication in the academic literature.

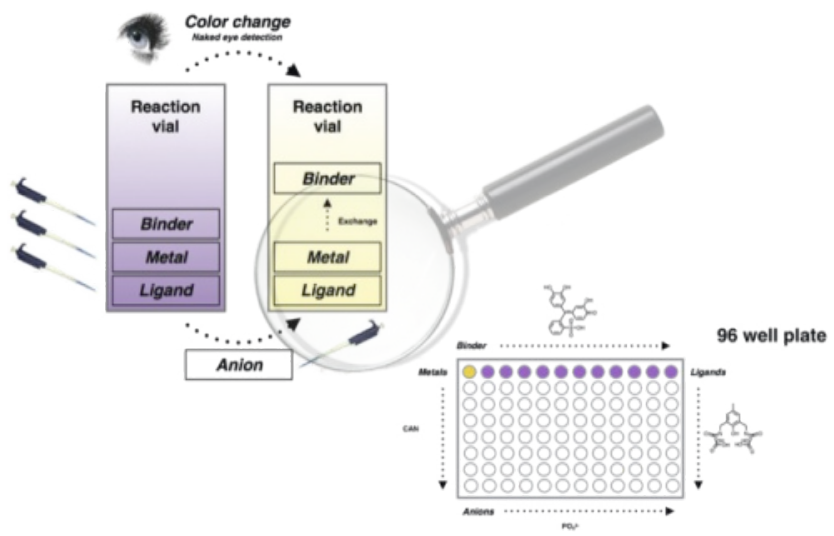

Fig. 1. Principle of the indicator displacement assay (IDA) for the recognition of analytes. A library of metals (salts) is mixed with a library of ligands (commercial) in a 96-well plate. The library of complexes is mixed with a library of indicators (binders). A visual and/or spectrophotometric measurement is carried out to demonstrate the formation of the coloured complexes formed. The library of complexes is then queried with an analyte library. The change of colour from blue to yellow (in the case of pyrocatecol violet as binder) is measured by visual and spectrophotometric detection. The detection system formed is then analysed for its analytical properties.

\section{Experimental Methodology}

The general methodology for the formation of analyte (such as anions) detectors with high-school students is as follows (Fig. 2). A library of $m$ commercial ligands is constituted (Fig. 3). In parallel, a library of $n$ metals (commercial salts) is constituted. A matrix of $n$ $\times m=C$ inorganic complexes is created and measured for its spectrophotometric properties in solution (buffers), visually and with a spectrophotometer in a 96-well-plate. The library of inorganic complexes $C$ is then exposed to a library of commercial indicators (Fig. 4) that bind with good affinity to the complexes and change colour upon binding. A library $n \times m \times 0=D$ is thus constituted, and the hits determined. The library $D$ is then subjected to reaction with a library of anions $p$ constituting a collection $E$, and the variations of spectrophotometric properties are measured between the library $D$ and $E$. Hits are further investigated for publication. As an example, a combination of 3 ligands with 3 metals, 3 indicators and 3 anions with 3 different buffers at 3 different $\mathrm{pH}$ allows the creation of a library of 729 possibilities, the equivalent of about 7.5 multiwell plates!

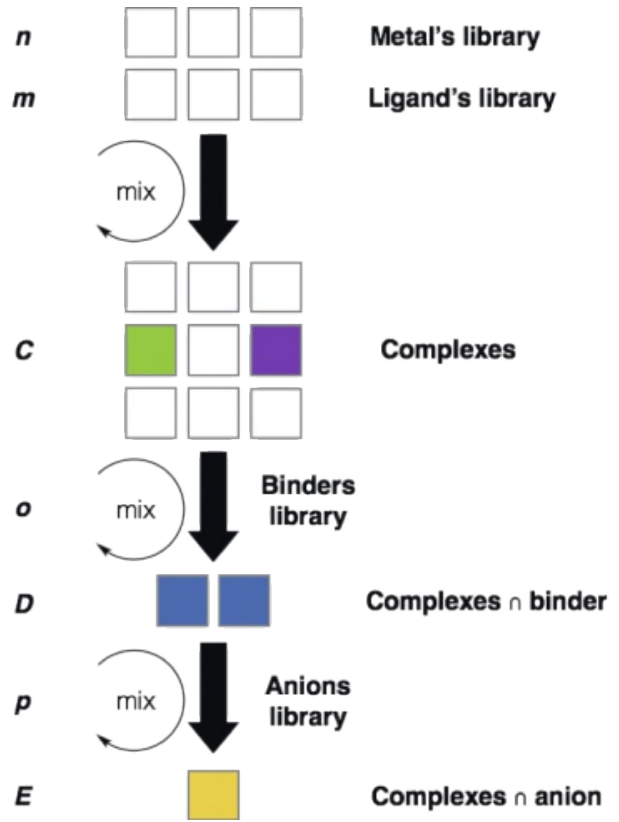

Fig. 2. A library of commercial metal precursors $(n)$ is mixed in a 96-well plate with a library of commercial ligands $(m)$. Formed complexes offer a library of complexes $(C)$. The identified complexes are mixed with a library of indicators (o) such as pyrocathecol violet. Formed coloured heteroleptic complexes $(D)$ are then measured for their spectrophotometric properties and exposed to a library of analytes $(p)$, forming a new library $(E)$. The $D$ and $E$ libraries are compared with the naked eye and with the spectrophotometer for variations in colours. If hits are obtained, they are further investigated.

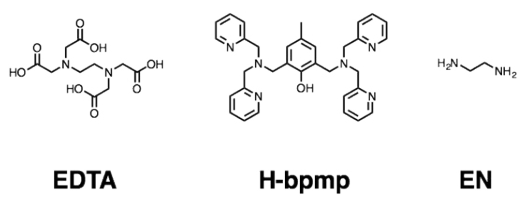

Fig. 3. Some commercial ligands that can be used for the creation of inorganic complexes in water.

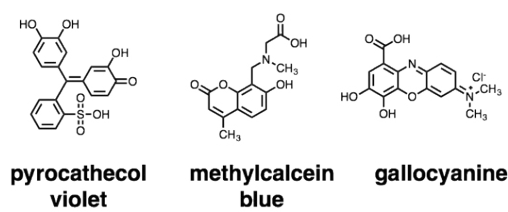

Fig. 4. Some commercial indicators that can be used as binders for the creation of IDAs. 


\section{Note:}

i) A spectrophotometer is not required for these measurements which can be made qualitatively with the naked eye for screening.

ii) If spectrophotometric measurements are required and equipment is not available, a scanner with a computer may be used instead of the spectrophotometer as suggested by Whitesides et al. ${ }^{[12]}$

\section{Results}

Each year, various research projects were investigated with students using the methodology presented here. In 2016, we decided to explore the possibility of phosphate detection using indicator displacement assays. The details of the project are given in section 4.1. In 2017, we extended the concept of detection with the creation of smart polymers. Details of the projects are given in section 4.2. In 2018, we explored the possibility of multi-detection such as the creation of molecular logic gates. We investigated the possibility of using nickel-based biosensors as presented in section 4.3. Finally, this year, we will present a poster at the SCS Fall Meeting about a simple fluorescent-based biosensor for detection of phosphate ions in water and how to use it for the detection of glyphosate (see section 4.4).

\subsection{A Cerium-based Indicator Displacement Assay for the Selective Naked-eye Detection of Phosphates}

In 2016, we created an indicator displacement assay (IDA) ${ }^{[13]}$ that was used to probe phosphate ions in water using a binuclear tems able to detect phosphates selectively and extract them out of water are urgently needed. In this spirit, we embedded an inorganic complex $[\mathrm{Ce}(\mathrm{PCV})]^{2+[14]}$ into gelatine (an easily accessible and inexpensive commercial polymer) to yield a Polymeric Indicator Displacement Assay (PIDA) (Fig. 6). The polymer changes colour from blue to yellow once exposed to a solution of phosphates, indicating their absorption, effectively soaking the phosphate out of solution. The polymer, extremely easy and inexpensive to assemble in high yield, is stable at room temperature and allows naked-eye detection of phosphate. We presented this research at the SCS Fall Meeting in 2017 as a poster in the Inorganic Chemistry session.

\subsection{A Molecular Logic Gate for the Naked-eye Detection of Phosphates or Thiol-based Molecules}

Thiols, containing molecules such as glutathione (GSH) or cysteine (Cys), are described in the literature as targeting metals. For example, GSH - a thiol-containing peptide - is produced in human cells for the detoxification of poisonous metals such as cadmium. Even if it is well accepted that GSH targets metals, the coordination modes are numerous and differ depending on the metal. Moreover, very few modes are reported for precious metals such as ruthenium or palladium. ${ }^{[15]}$ Here, we showed that nickel complexes, bearing pyrocathecol violet (PCV) as a ligand, can be easily formed in buffered conditions (Fig. 7). [14] These blue nickel complexes can be scrutinized using a so-called Indicator Displacement Assay (IDA) to recognize phosphate or thiol-containing molecules such as pyrophosphate or GSH/Cys.
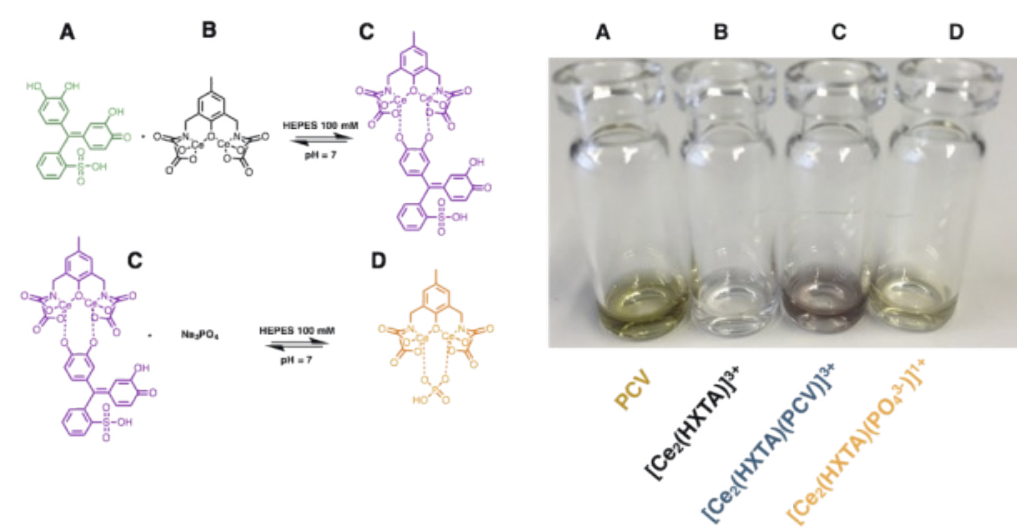

Fig. 5. Naked-eye detection of $\mathrm{PO}_{4}^{3-}$ : The assay is based on the violet coloured $\left[\mathrm{Ce}_{2}(\mathrm{HXTA})(\mathrm{PCV})\right]^{+} \mathrm{C}$, which upon addition of $\mathrm{PO}_{4}{ }^{3-}$, changes colour to yellow D. B Is a $250 \mu \mathrm{M}$ solution of $\left[\mathrm{Ce}_{2}(\mathrm{HXTA})\right]^{3+}$ in aqueous buffer (HEPES $100 \mathrm{mM}, \mathrm{pH} 7.4$ ) solution. The colour of the pyrocathecol violet A changes from yellow $\left(\lambda_{\max }=445 \mathrm{~nm}\right)$ to violet $\left(\mathrm{C} \lambda_{\max }=580 \mathrm{~nm}\right)$ upon formation of [Ce $\left.{ }_{2}(\mathrm{HXTA})(\mathrm{PCV})\right]^{+}$. Addition of phosphate anion $(2500 \mu \mathrm{M})$ displaces pyrocathecol violet $\left[\mathrm{Ce}_{2}(\mathrm{HXTA})(\mathrm{PCV})\right]^{+}$to form a pale yellow solution $\mathrm{D}$.

complex $\left[\mathrm{Ce}_{2}(\mathrm{HXTA})\right]^{3+}$ (Fig. 5). The homoleptic complex is able to detect phosphate ions at micromolar concentrations both spectrophotometrically and with the naked eye. Pyrocathecol violet, ${ }^{[6]}$ a $\mathrm{pH}$-sensitive dye, is exchanged upon addition of phosphate on $\left[\mathrm{Ce}_{2}(\mathrm{HXTA})(\mathrm{PCV})\right]^{+}$allowing a colour change from blue-violet to yellow at micromolar concentrations. Our system has the best affinity constant of the literature to date compared to similar systems, up to $\mathrm{K}_{\text {affinity }}=10^{7} \mathrm{M}^{-1}$. We presented this research at the SCS Fall Meeting in 2016 as a poster in the Inorganic Chemistry session.

\subsection{A Simple and Smart Colour-change Bioinorganic Polymer for the Naked Eye and Absorption of Phosphates in Water}

High concentrations of phosphates in water are characteristic of eutrophication of fresh water systems, causing severe negative ecological impacts in our lakes and rivers. Therefore, simple sys- a)

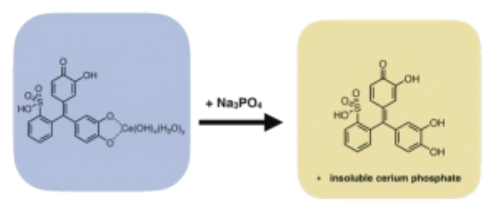

b)

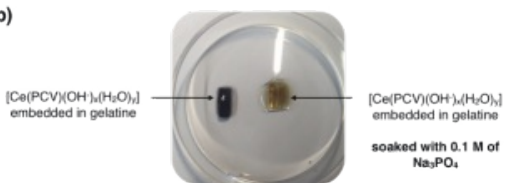

Fig. 6. Principle of the PIDA. a) $\left[\mathrm{Ce}(\mathrm{IV})(\mathrm{PCV})\left(\mathrm{OH}^{-}\right)_{x}\left(\mathrm{H}_{2} \mathrm{O}\right)_{y}\right](250 \mu \mathrm{M}) \mathrm{em}-$ bedded in gelatine (HEPES $100 \mathrm{mM} \mathrm{pH}=7.4$ ) forms [Ce(IV)(PCV)(OH $)_{x}$ $\left.\left(\mathrm{H}_{2} \mathrm{O}\right)_{y}\right] \subset$ gelatine, a blue polymer, which once exposed to phosphate $(0.1 \mathrm{M})$ changes colour from blue to yellow forming cerium phosphate embedded in gelatine. b) Macroscopic view of the blue [Ce(IV)(PCV)(OH') $\left.{ }_{x}\left(\mathrm{H}_{2} \mathrm{O}\right)_{y}\right] \subset$ gelatine and the yellow product after soaking in $0.1 \mathrm{M}$ of sodium phosphate in a $100 \mathrm{mM}$ HEPES $\mathrm{pH}=7.4$. 
The PCV ligand forming the Ni-complex is also a 1,4-Michael acceptor, therefore a competition with an indicator displacement and a 1,4-Michael addition (1,4-MA) for recognition may take place. We showed that, for both cysteine and L-reduced GSH, the 1,4-MA is surprisingly preferred to the IDA, suggesting that thiol-containing molecules are more likely to engage a 1,4-MA than a ligand exchange, even in presence of a metal. When in the presence of pyrophosphate, the indicator displacement assay is preferred. The one-complex two-mechanism possible reaction gives birth to a molecular two-output signal Boolean logic gate. We presented this research at the SCS Fall Meeting in 2018 as a poster in the Inorganic Chemistry session.

\subsection{A Simple Fluorescent-based Indicator Displacement Assay for the Selective Naked-eye Detection of Phosphates}

A simple fluorescent indicator displacement assay (IDA) was used to probe phosphate ions into water by mixing only methylcalcein blue (MCB) and cerium ammonium nitrate (CAN) (Fig. $8)$. The fluorescence of MCB is quenched when added to CAN ${ }^{[16]}$ in buffered conditions (HEPES $100 \mathrm{mM}, \mathrm{pH}=7.4$ ) and recovered by the addition of phosphate due to ligand exchange. Compared to previous results presented in $2016^{[17]}$ (see section 4.1), here we a)

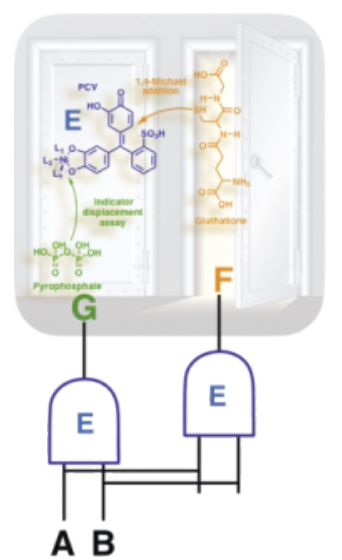

b)

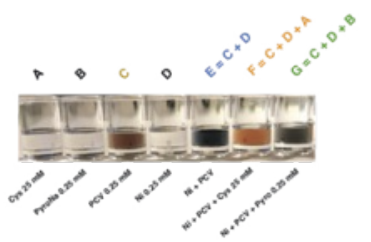

Fig. 7. Mixing $\mathrm{NiSO}_{4}$ with PCV (HEPES $100 \mathrm{mM} \mathrm{pH}=7.4$ ) produces [Ni(PCV)] $(250 \mu \mathrm{M})$ a blue-coloured water-soluble inorganic complex $\mathrm{E}$. This inorganic complex $E$ is exposed to the presence of either sodium pyrophosphate $\mathrm{G}(2500 \mu \mathrm{M})$ or cysteine (or gluthatione) $(2500 \mu \mathrm{M}) \mathrm{F}$. Upon exposure to sodium pyrophosphate $\mathrm{G}$, the complex $\mathrm{E}$ changes colours to green through an indicator displacement assay, whereas upon exposition to cysteine $F$, the complex $E$ changes colours to orange $F$, suggesting a 1,4-Michael addition. show that this simple homoleptic complex is able to detect selectively phosphate ions below micromolar concentrations both spectrophotometrically and with the naked eye. In addition, we will also present our efforts towards the detection of glyphosate.

\section{Conclusion}

In this pioneering project 'La Chimie en Couleurs', we aimed to demonstrate that:

- Students can experience science as a colourful activity full of exciting and unpredictable results.

- Real research can be practiced at high-school level giving new, valuable scientific data.

- IDAs are suitable for research at high-school level, leading to original, visually impactful (colourful) but analytically meaningful results, using a simple setup and inexpensive reagents.

- A simple and 'standardized' methodology can be implemented to achieve 'quick' experimental results using IDAs.

- Our high-throughput screening protocol of IDAs offers a rich and virtually unlimited source of potentially useful data.

- Science carried out at a high school can compete with more advanced academic research, such as carried out by $\mathrm{PhD}$ students and postdocs (SCS poster prize 2016).

\section{Perspectives}

In the future, we would like to:

- Target each maturity project toward one type of recognition (anions, sugars, small molecules, proteins, etc.).

- Create an online database with all the results.

- Extend research to include research stays at laboratories of different academic mentors and/or different high schools, thus creating larger amounts of data.

- Generate big data analysis with this database that could create new insight into the field of bio-sensing and open new research perspectives.

- Spread the idea that high school students can contribute significantly to scientific research in a fun and original way.

- Increase the exposure of students to the academic community, for example by allowing more students to visit academic labs or to participate in scientific meetings.

- Ultimately, publish our methodologies and research results in prestigious academic journals. ${ }^{[18]}$

\section{Acknowledgements}

The authors of the present article would like to thank the Gymnase français de Bienne for providing great support in carrying out this project, in particular Christine Gagnebin and Pierre-Etienne Zürcher (Rectrice et Recteur). We also would like to thank the EMSp direction, including Yves Pillard and Dr. Mathieu Aurousseau, for unconditional support and patience in helping doing science with the students, and for

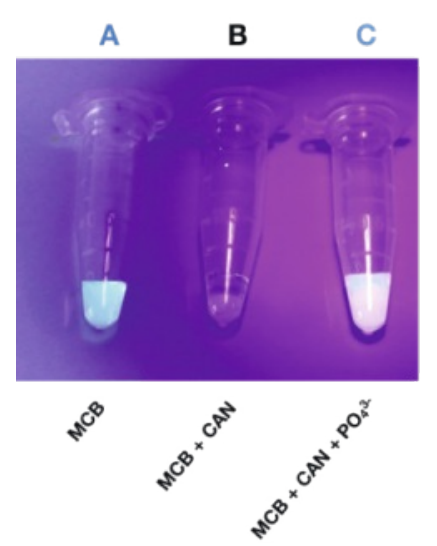

A
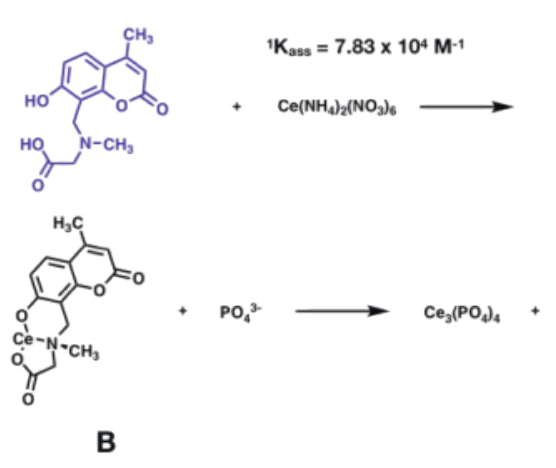
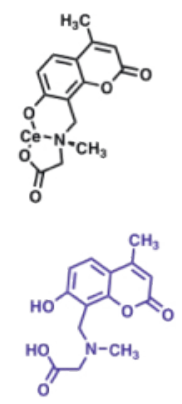

C

Fig. 8. Mixing CAN $(250 \mu \mathrm{M})$ with MCB $(250 \mu \mathrm{M})$ A offers [Ce(MCB)] ${ }^{2+} \mathrm{B}(250 \mu \mathrm{M})$ a non-fluorescent water soluble inorganic complex. This inorganic complex $B$ is exposed to the presence of anions. Upon exposure to sodium phosphate $(2500 \mu \mathrm{M})$, the complex B disassembles via an indicator displacement assay producing fluorescence visible with the naked eye under the UV lamp at 254 and $400 \mathrm{~nm}$. 
the help with the lab renovation. Gratitude goes also to Prof. Dr. Thomas $\mathrm{R}$. Ward for support in setting up a functional research lab by providing equipment such as a spectrophotometer and a pipetting station. Finally, the authors would like to thank all the students, who have shown so much enthusiasm for science, and trust in their mentors, such as Bruno Cabete, Liam McGillivray, Anthony Racine, Giuseppe Giordano, Mugé Ozkül, Caroline Schneider, Anais Christen.

Received: June 3, 2019

[1] K. P. C. Vollhardt, 'Traité de chimie organique', Bruxelles: De BoeckWesmael S. A., 1995.

[2] C. Housecroft, A. G. Sharpe, 'Inorganic Chemistry', Madrid: Pearson, 2005.

[3] R. Noyori, Angew. Chem. Int. Ed. 2002, 41, 2008.

[4] C. K. Jorgensen, 'Inorganic Complexes', London \& New York: Academic Press, 1963

[5] N. K. Terrett, 'Combinatorial Chemistry', Oxford, New York, Tokyo: Oxford University Press, 1998.
[6] M. S. Han, D. H. Kim, Angew. Chem. Int. Ed. 2002, 41, 3963.

[7] 'Synthetic Receptors for Biomolecules, Design Principles and Applications', Ed. B. D. Smith, Indiana USA, 2015.

[8] K. Severin, Curr. Opin. Chem. Biol. 2010, 14, 737.

[9] S. L. Wiskur, H. Ait-Haddou, J. J. Lavigne, E. V. Anslyn, Acc. Chem. Res. 2011, 32, 963.

[10] W. Yu, J. Qiang, J. Yin, S. Kambam, F. Wang, Y. Wang, X. Chen, Org. Lett. 2015, 16, 2220.

[11] B. T. Nguyen, E. V. Anslyn, Coord. Chem. Rev. 2006, 250, 3118.

[12] D. C. Christodouleas, A. Nemiroski, A. A. Kumar, G. M. Whitesides, Anal. Chem. 2015, 87, 9170.

[13] M. E. Branum, A. K. Tipton, S. Zhu, L. Que, J. Am. Chem. Soc. 2001, 123, 1898.

[14] K. Macek, L. Moravek, Nature 1956, 178, 102.

[15] B. K. Bibhesh, Asian J, Chem. 2005, 17, 1.

[16] H. G. Britain, Anal. Chem. 1987, 59, 1122.

[17] T. Rossel, M. Creus, ChemRxiv 2018.

[18] https://www.sciencematters.io. 